\title{
Enhancement of nonlinear optical phenomena by localized resonances
}

\author{
A. Rodríguez Echarri, ${ }^{\dagger}$ Joel D. Cox, ${ }^{\dagger}$ Renwen $\mathrm{Yu}^{\dagger}$ and \\ F. Javier García de Abajo*,†, \\ $\dagger$ †CFO-Institut de Ciencies Fotoniques, The Barcelona Institute of Science and Technology, \\ 08860 Castelldefels (Barcelona), Spain \\ ¥CREA-Institució Catalana de Recerca i Estudis Avançats, Passeig Lluís Companys 23, \\ 08010 Barcelona, Spain \\ E-mail: javier.garciadeabajo@nanophotonics.es
}

\begin{abstract}
Nonlinear optics at the nanoscale is severely limited by the small departure of available materials from linear behavior. Despite intense efforts placed into overcoming this problem using multiple strategies for enhancing the near-field light intensity, all-optical active nanodevices remain a challenge. Here we introduce a material-independent scheme for quantifying the enhancement of the nonlinear response in nanostructures assisted by proximal metallic or dielectric nanoresonators. The proposed figures of merit, which we apply to configurations of current interest incorporating 2D materials and dielectric cavities, can be generally used to optimize nonlinear nanoscale elements.
\end{abstract}

Keywords: surface plasmons, nonlinear optics, electromagnetic modeling, integrated optics, harmonic generation

The control of light by light based on nonlinear optical phenomena has led to significant technological advances in photonics, ${ }^{1,2}$ particularly regarding communications, ${ }^{3}$ biological 
imaging, ${ }^{4-8}$ light modulation, ${ }^{9-12}$ and THz sources. ${ }^{13}$ The challenge of nonlinear optics stems from the negligible interaction among electromagnetic waves in free space. Substantial efforts have been devoted to identify materials with sufficient anharmonic response as to enable optical nonlinearities at low light powers. ${ }^{14-17}$ Unfortunately, the best currently available nonlinear materials exhibit only a weak response, which needs to be compensated in practical devices by for example making light pass through long paths in bulk crystals, a strategy that demands stringent phase-matching conditions. ${ }^{10,18,19}$ Additionally, near-field intensity enhancement through optical resonators has been widely explored to design active photonic devices operating on subwavelength scales for applications in optical circuitry, ${ }^{20,21}$ biological sensing, ${ }^{7,8,22-24}$ and quantum information. ${ }^{25-27}$

Nanoscale nonlinear optics has received a recent stimulus with the isolation of graphene ${ }^{28}$ and other atomically-thin crystals, ${ }^{29-31}$ which combine a large electro-optical response with strong intrinsic optical nonlinearities. In particular, the conical electronic dispersion of graphene boosts its nonlinear response through both intra- and interband transitions, ${ }^{12,32,33}$ which are predicted to be further increased by coupling to plasmons -the collective oscillations of electrons in conducting media- sustained by highly doped graphene nanostructures. ${ }^{34-36}$ Also, transition-metal dichalcogeneides such as $\mathrm{MoS}_{2}$ are observed to produce efficient harmonic generation. ${ }^{37-42}$

Despite their large nonlinear optical susceptibilities, the response of 2D materials is limited by their inherently small volumes. Optical resonances provide a promising way for circumventing this limitation, as they can enhance the driving electric field, thus boosting the effective nonlinear response. This idea has been generically pursued with nonlinear materials using plasmons to concentrate electromagnetic energy down to extremely subwavelength volumes ${ }^{43-45}$ Mie-like resonances in dielectric cavities, ${ }^{46}$ and lattice resonances in periodic structures. ${ }^{16,17}$

In this article we explore the enhancement of the nonlinear optical response that can be achieved in 2D materials by means of resonant cavities. Specifically, we consider (1) the 
extrinsic near-field enhancement provided by localized nanoparticle plasmons acting on a 2D material; (2) the extrinsic enhancement in a 2D material produced by Mie-like resonances in dielectric nanoparticles; (3) the intrinsic enhancement of optical nonlinearity in a dielectric nanoparticle by its supported Mie resonances; (4) the effect of lattice resonances on the nonlinear response for a periodic 2D array of dielectric nanodisks. This suite of different resonant cavities are examples that cover a wide range of possibilities: plasmonic nanoparticles, represented by silver nanorods (a more detailed analysis could explore the effect of material and morphology, using for example modal expansions ${ }^{47}$ or quasi-normal modes ${ }^{48}$ ); dielectric nanocavities, represented by silicon spheres and $\mathrm{LiNbO}_{3}$ disks (other dielectric materials could be used, as well as engineered geometries ${ }^{49-51}$ ); and lattice resonances in $\mathrm{LiNbO}_{3}$ disk arrays (there is extensive literature on the field enhancement caused by such resonances ${ }^{52}$ ). We focus in particular on the enhancement in second- and third-harmonic generation, as well as the Kerr nonlinearity, quantifying the yield of these nonlinear processes in each of the systems studied.

\section{Enhancement of nonlinear optical phenomena by reso-}

\section{nant cavities}

The nonlinear response associated with an $n^{\text {th }}$-order process generating harmonic $s$ of the fundamental frequency $\omega$ can be quantified by the associated induced dipole moments $p_{s \omega}^{(n)}$, which we write below for second-harmonic generation (SHG, $n=s=2$ ), third-harmonic generation (THG, $n=s=3$ ), and the Kerr nonlinearity $(n=3, s=1)$, respectively. We consider systems for which emission from the nonlinear dipole moments dominates the response in the far field, although in principle multipolar moments can contribute significantly when the particle size is commensurate with the incident wavelength. The corresponding induced dipoles $\mathbf{p}_{s}^{(n)}$ are related to the self-consistent near electric field $\mathbf{E}(\mathbf{r}, t)=\mathbf{E}(\mathbf{r}) \mathrm{e}^{-\mathrm{i} \omega t}+$ c.c. 
and the nonlinear susceptibilities $\chi_{s}^{(n)}$ as

$$
\begin{aligned}
p_{2 \omega, i}^{(2)} & =\sum_{j k} \int_{V} d^{3} \mathbf{r} \chi_{2 \omega, i j k}^{(2)} E_{j} E_{k}, \\
p_{3 \omega, i}^{(3)} & =\sum_{j k l} \int_{V} d^{3} \mathbf{r} \chi_{3 \omega, i j k l}^{(3)} E_{j} E_{k} E_{l}, \\
p_{\omega, i}^{(3)} & =\sum_{j k l} \int_{V} d^{3} \mathbf{r} \chi_{\omega, i j k l}^{(3)} E_{j} E_{k} E_{l}^{*},
\end{aligned}
$$

where the $i-l$ indices denote Cartesian components, and the integration in Eqs. (1) takes place within the volume $V$ of a nonlinear material. For the sake of generality, we express the nonlinear susceptibility tensors in the form $\chi_{2 \omega, i j k}^{(2)}=\chi_{2 \omega}^{(2)} \Delta_{i j k}^{(2)}$ for SHG, and $\chi_{s \omega, i j k l}^{(3)}=\chi_{s \omega}^{(3)} \Delta_{i j k l}^{(3)}$ for third-order processes. In this manner the frequency-dependent properties of a material are contained in the scalar quantities $\chi_{s \omega}^{(n)}$, while the crystal symmetries are encoded in the $\Delta_{i j k}^{(n)}$ tensors that determine the dependence of $p_{s \omega}^{(n)}$ on the driving electric field within this perturbative description. Then, from Eqs. (1), we define the normalized nonlinear dipole strength, which we use as a figure of merit (FoM) to quantify the enhancement of nonlinear processes:

$$
\mathcal{F}_{s \omega}^{(n)} \equiv \frac{1}{V\left|E^{\text {inc }}\right|^{n}}\left\|\frac{\mathbf{p}_{s \omega}^{(n)}}{\chi_{s \omega}^{(n)}}\right\| .
$$

Here the double bars indicate the norm of the vector quantity therein and $E^{\text {inc }}$ is the amplitude of the impinging electric field, which is assumed to be a plane wave. By normalizing to the scalar part of the nonlinear susceptibility along with the electric field, the FoM provides a material-independent (note that $\mathbf{p}_{s \omega}^{(n)} \propto \chi_{s \omega}^{(n)}$ ) quantitative analysis of the enhancement in the nonlinear response relative to that of the system interacting only with the driving external field. Other FoMs have been defined for nonlinear processes, ${ }^{45,53,54}$ but the above definition is particularly simple and generally suitable for different orders.

Note that while Eqs. (1) and (2) are given for 3D media, they can be used to describe nonlinear enhancement in 2D media by making the following two changes in Eqs. (1) and 
(2): $d^{3} \mathbf{r} \rightarrow d^{2} \mathbf{R}$, where $\mathbf{R}=(x, y)$ denotes the position vector in the $z=0$ plane that the 2D material occupies; and $V \rightarrow A$, where $A$ denotes the integration area occupied by the material. The integration area is determined by the extension of both the $2 \mathrm{D}$ material and the optical modes of the resonant cavity, which can be problematic because in open systems the latter extend over the entire space. For convenience, we adopt here simple prescriptions consisting in restricting the integration area to either the projected area of the resonating particle or a multiple of it (see below), where the dominant near-field contribution is expected to occur, although a more rigorous prescription could be formulated in terms of the well-established quasi-normal modes. ${ }^{48}$ In contrast, for nonlinear 3D particles, the volume is simply restricted by that of the cavity.

In what follows, we study the second- and third-order nonlinear response of $2 \mathrm{D}$ materials exposed to optical resonances. Keeping in mind that second-order effects are prohibited in centrosymmetric media, we address them by focusing on a particular case of materials without inversion symmetry: we adopt the second-order tensor corresponding to the $D_{3 h}$ point symmetry group ${ }^{10}$ (i.e., $\Delta_{x x x}^{(2)}=-\Delta_{x y y}^{(2)}=-\Delta_{y y x}^{(2)}=-\Delta_{y x y}^{(2)}$ ), which describes for example transition-metal dichalcogeneides of the form $\mathrm{MX}_{2}$ with $\mathrm{M}=\mathrm{Mo}$ or $\mathrm{W}$ and $\mathrm{X}=\mathrm{S}$, Se, or Te. Conversely, third-order phenomena can be realized in isotropic 2D media such as graphene, characterized by the tensor $\Delta_{i j k l}^{(3)}=\delta_{i j} \delta_{k l}+\delta_{i k} \delta_{j l}+\delta_{i l} \delta_{j k} \cdot{ }^{10}$ From this consideration, we find that in the 2D material structures under study enhancement in the nonlinear response occurs when $\mathcal{F}_{2 \omega}^{(2)}>4 \sqrt{2}$ or $\mathcal{F}_{s \omega}^{(3)}>6 \sqrt{2}$ for second- and third-order processes, respectively, while in isotropic $3 \mathrm{D}$ materials, $\mathcal{F}_{s \omega}^{(n)}>1$ suffices.

\section{RESULTS AND DISCUSSION}

Plasmonically-enhanced nonlinear response in 2D materials. We first investigate the enhancement of the nonlinear response produced in a 2D material by the near-field associated with localized plasmons excited in a neighboring noble metal nanoparticle. This subject 


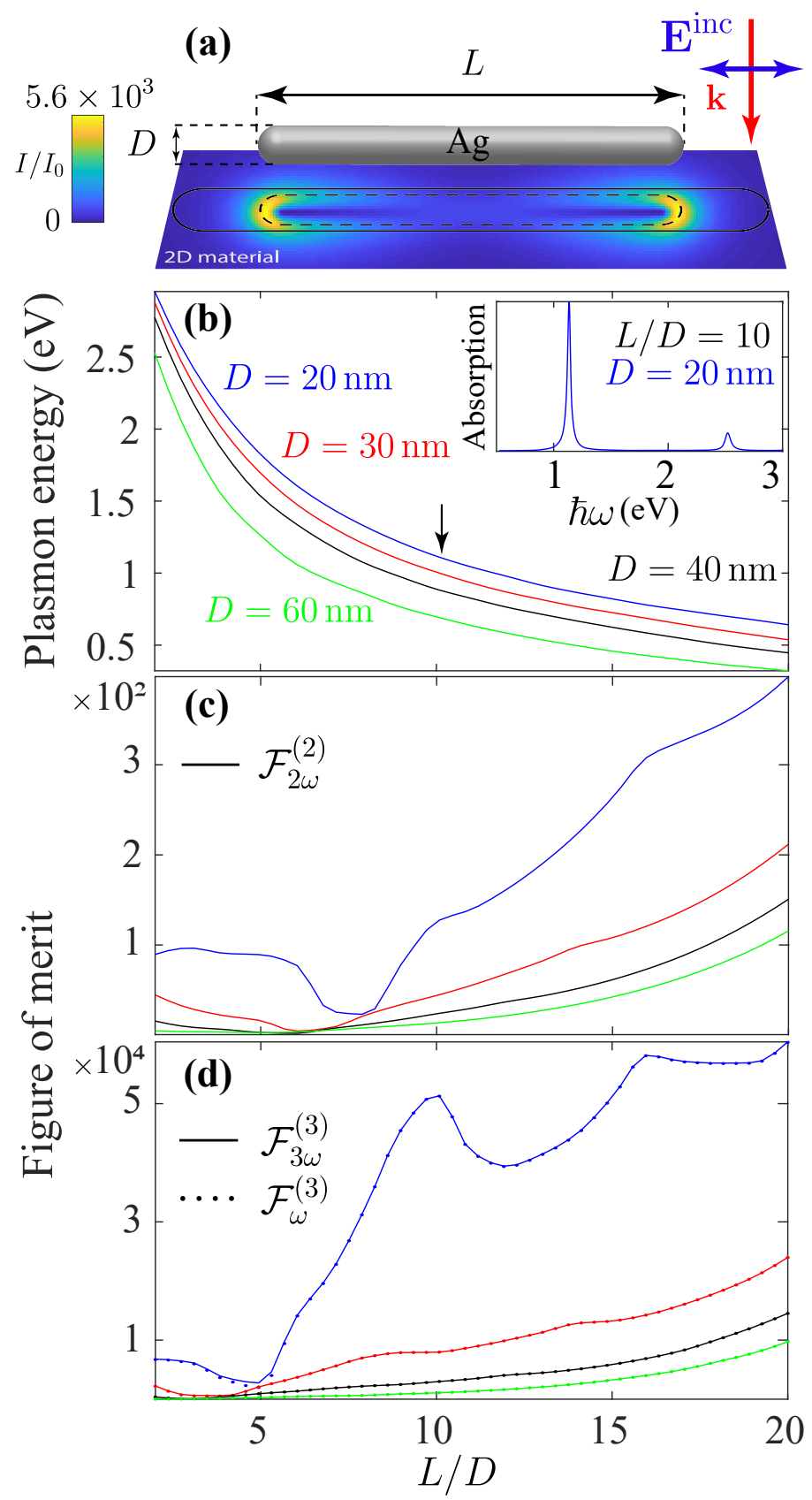

Figure 1: Extrinsic plasmonic enhancement of nonlinear optical phenomena in 2D materials by noble metal nanostructures. (a) Plasmons in a silver nanowire placed $1 \mathrm{~nm}$ above a 2D material (color map plane) are excited by a normally-incident light plane wave (wave vector $\mathbf{k}$ ) of electric field $\mathbf{E}^{\text {inc }}$ parallel to the nanowire, producing strong local electric fields, as shown in the color map, where the 2D material is assumed to be placed. A dashed curve shows the profile of the wire projected on the 2D material plane, while a solid curve indicates the integration area. (b) Energy of the lowest-order dipolar plasmon as a function of the nanowire length-to-diameter ratio $L / D$ for different $D$ 's. The inset shows an absorption spectrum for $L / D=10$ and $D=20 \mathrm{~nm}$. (c,d) Nonlinear FoM (see Eq. (2)) for SHG (c), as well as THG and the Kerr nonlinearity (d), for the wires of (b). 
has been extensively discussed in the literature, ${ }^{43-45}$ with particular emphasis on glasses incorporatig metallic inclusions, ${ }^{10}$ so we only present an illustrative example consisting of a silver nanowire (i.e., a combination of a good plasmonic metal and a resonating geometry) deposited on the 2D material surface (1 nm surface-to-surface separation), as shown in Fig. 1a. We simulate the extinction and near field in this system using the boundary-element method (BEM), ${ }^{55}$ with the measured permittivity of silver as input. ${ }^{56}$ In particular, we study the dependence on the length $L$ and diameter $D$ of the wire when the system is illuminated by light tuned to the lowest-order dipole plasmon. The intensity distribution of the induced electric field in the plane of the 2D material is indicated by the color map, showing intensity levels exceeding the incident intensity by four orders of magnitude for a wire length of $200 \mathrm{~nm}$ and a width of $20 \mathrm{~nm}$, which are realistic parameters achievable through colloid chemistry. ${ }^{57}$ The plasmon resonance, which manifests as a prominent peak in the extinction cross-section $\sigma^{\text {ext }}$ (see Fig. 1b inset), can be tuned within a broad range of photon energies by changing the wire geometry, as we show in Fig. 1(b) for a selection of particle diameters. The plasmon resonance is observed to redshift in general as either the length or the width of the nanowire is increased. ${ }^{58}$

Using Eq. (2), we quantify the plasmon-enhanced nonlinear response of the 2D material in the presence of the $\mathrm{Ag}$ nanowire by taking the effective area $A$ as twice that projected by the particle onto the $2 \mathrm{D}$ material plane (i.e., the area contained within the solid curve of Fig. 1a, which is obtained by scaling the projected profile proportionally). Note that we are neglecting the modification produced in the plasmonically-enhanced electric field as a result of the interaction with the $2 \mathrm{D}$ material, which is a reasonable assumption for nonresonant atomically-thin layers. We plot the nonlinear FoM in Fig. 1c for SHG and in Fig. 1d for THG and the Kerr nonlinearity. Remarkably, we observe plasmon-enhancement factors of the order of $10^{4}$ and $10^{6}$ for second- and third-order processes, respectively. As a rule of thumb, we find that higher aspect ratios $L / D$ produce larger overall nonlinear enhancements when normalized to the projected wire area. 
(a)

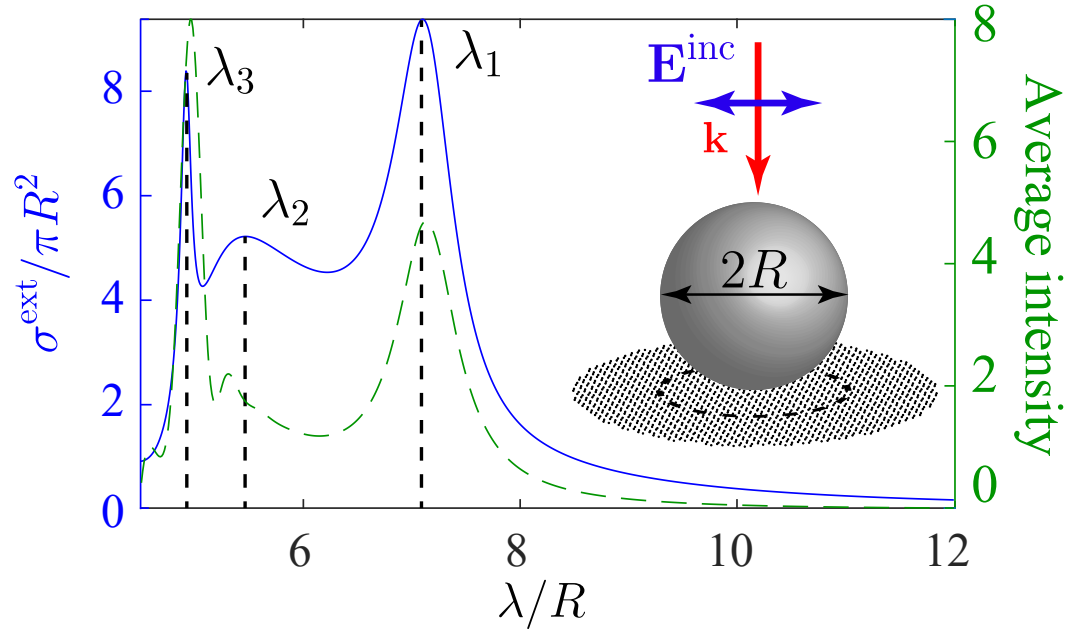

(b)

\begin{tabular}{c|c|c|c}
\hline & $\lambda_{1}$ & $\lambda_{2}$ & \multicolumn{1}{c}{$\lambda_{3}$} \\
\hline $\mathcal{F}_{2 \omega}^{(2)}$ & 4.3 & 1.5 & 10.0 \\
$\mathcal{F}_{3 \omega}^{(3)}$ & 31.2 & 6.5 & 130.4 \\
$\mathcal{F}_{\omega}^{(3)}$ & 32.3 & 7.7 & 131.2 \\
\hline
\end{tabular}

Figure 2: Nonlinear optical enhancement by Mie resonances. (a) We show the extinction cross-section of a Si sphere normalized to its projected area as a function of light wavelength normalized to the sphere radius $R$ (solid curve) compared with the average of the in-plane electric field intensity over the circle projected by the sphere (area inside the dashed curve of the inset). Various resonances are identified as magnetic $\left(\lambda_{1}\right.$ and $\left.\lambda_{3}\right)$ and electric $\left(\lambda_{2}\right)$. (b) Tabulated nonlinear FoM for several nonlinear processes using the resonances identified in (a) over the projected area (see Eq. (2)).

Enhancement of 2D-material nonlinearities by Mie resonances. Mie resonances emerge as a result of optical confinement in dielectric structures, essentially driven by the affinity of the electromagnetic field for regions with higher permittivity. ${ }^{59}$ These types of modes require the size of the structure to be commensurate with the light wavelength inside the material. A dielectric sphere is a prototypical example of this phenomenon, admitting an analytical solution. ${ }^{60}$ Following the procedure outlined in the previous section to quantify the enhancement of the nonlinear response by plasmonic nanostructures, we investigate the enhancement in a 2D material by a contacting $\mathrm{Si}$ sphere of radius $R$. Note that for the wavelengths considered, Si has a relatively uniform and high refractive index $\approx 3.4$, so 
the Mie resonances are well-characterized, particularly for low-order magnetic modes. ${ }^{61} \mathrm{We}$ present the extinction cross-section $\sigma^{\text {ext }}$ and the linear field-intensity enhancement of the $\mathrm{Si}$ sphere in Fig. 2a, where the resonances are indicated by vertical dashed lines, along with a schematic illustration of the system under consideration. The indicated peaks correspond to magnetic $\left(\lambda_{1}\right.$ and $\left.\lambda_{3}\right)$ and electric $\left(\lambda_{2}\right)$ resonances. The values of the FoM associated with each resonance for the various nonlinear optical processes under consideration are tabulated in Fig. 2b, taking the effective area $A$ as that projected by the sphere onto the $2 \mathrm{D}$ material plane (dashed curve in inset of Fig. 2a). We find that magnetic resonances $\left(\lambda_{1}\right.$ and $\left.\lambda_{3}\right)$ generate large enhancement factors compared with the electric one $\left(\lambda_{2}\right)$. Interestingly, the FoM increases with multipolar order (dipolar in $\lambda_{1}$ and quadrupolar in $\lambda_{3}$ ), presumably as a result of the lower radiative broadening, and we note that this effect cannot be anticipated by only examining the associated cross-section. An interesting possibility consists in engineering the geometry to overlap a magnetic and an electric resonance in frequency as a way to further enhance the field.

Intrinsic nonlinear enhancement in a dielectric nanodisk. We now consider the effect of geometrical resonances in a dielectric nanostructure on its intrinsic nonlinear optical response, which has been previously studied for THG. ${ }^{50}$ Instead, we concentrate on SHG. As illustrated schematically in Fig. 3a (inset), we study a nanodisk of diameter $D$ and thickness $t$ illuminated by linearly-polarized light impinging under normal incidence with respect to the plane of the disk. The nanodisk is made of $\mathrm{LiNbO}_{3}$, which offers a comparatively high second-order response. ${ }^{62,63}$ We simulate the linear response using the BEM, adopting a refractive index of 2.25 for the material, as appropriate within the near-infrared spectral range. The extinction cross-section $\sigma^{\text {ext }}$, normalized to the projected area $A=\pi D^{2} / 4$, is presented in Fig. 3a as a function of light wavelength $\lambda$ (normalized to $D$ ) for three different aspect ratios: $D / t=1,2$, and 5 (color-coded curves). The cross section is found to exceed the disk area by a moderate factor, which actually decreases with increasing aspect ratio $D / t$ (i.e., for thinner disks); this result is expected, as the mode confinement is reduced when 


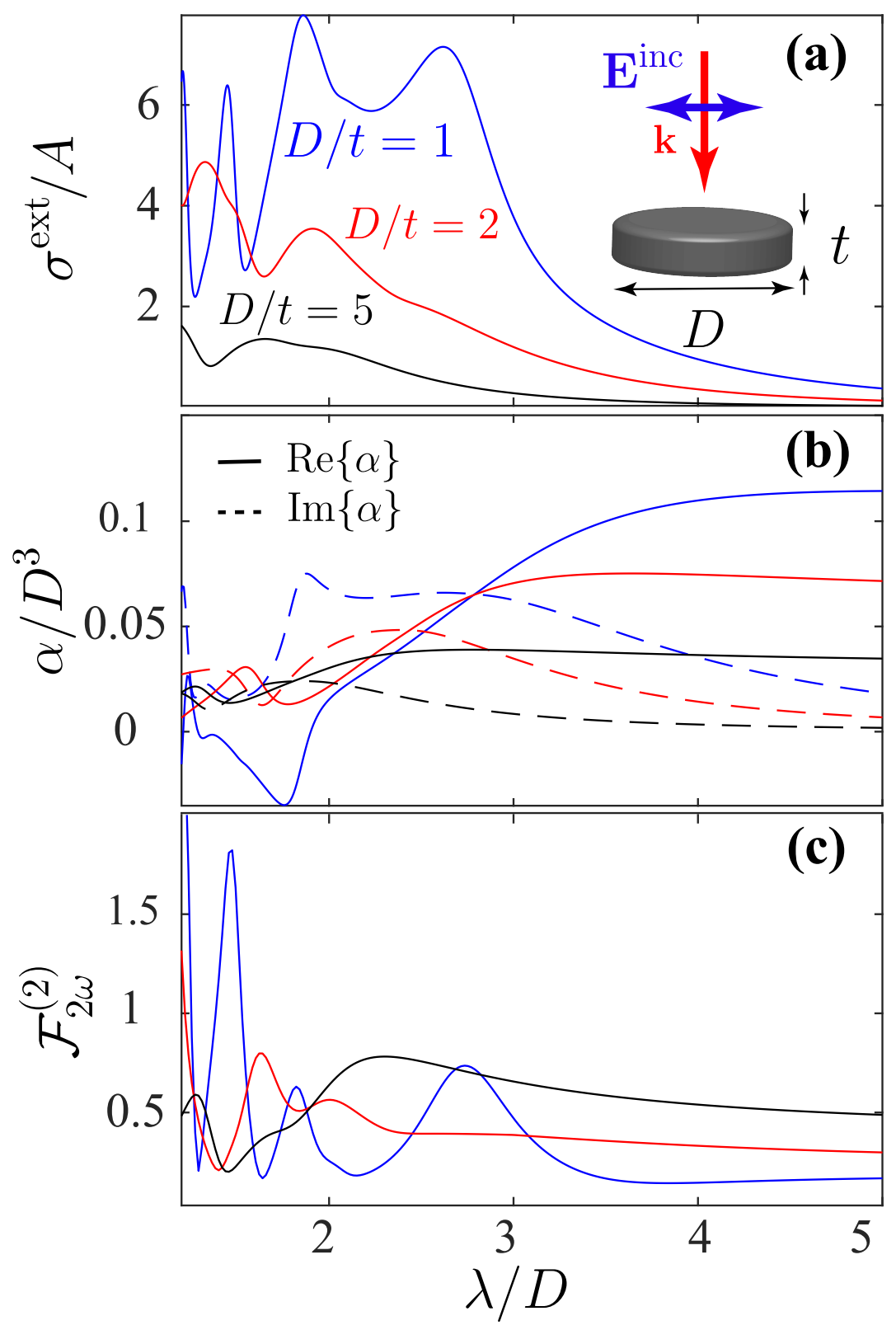

Figure 3: Linear and SHG response of a $\mathrm{LiNbO}_{3}$ disk. (a) We consider the system schematically illustrated in the inset, consisting of a $\mathrm{LiNbO}_{3}$ disk of diameter $D$ and thickness $t$. The main panel shows the extinction cross-section normalized to the disk cross-sectional area as a function of $\lambda / D$ for different values of the aspect ratio $D / t$. (b,c) Linear polarizability $\alpha_{\omega}^{(1)}$ normalized to $D^{3}$ (b) and nonlinear SHG figure of merit (c) for the color-coded aspect ratios considered in (a). 
the disk is thinned, thus increasing radiative damping, and therefore producing broader resonances. Similar conclusions are obtained by studying the disk polarizability $\alpha_{\omega}$ (Fig. $3 \mathrm{~b}$ ), yielding the induced dipole $\mathbf{p}_{\omega}^{(1)}=\alpha_{\omega} \mathbf{E}^{\text {inc }}$ in response to an incident field $\mathbf{E}^{\text {inc }}$ as a function of incidence frequency $\omega$. In practice, we calculate $\alpha_{\omega}$ by simulating the forward far-field amplitude $\mathbf{f}_{\omega}=(\omega / c)^{2} \alpha_{\omega} \mathbf{E}^{\text {inc }}$. Incidentally, from the optical theorem we know that the extinction cross-section (Fig. 3a) is simply given by $\sigma^{\text {ext }}=(4 \pi \omega / c) \operatorname{Im}\left\{\alpha_{\omega}\right\}$. We make further use of $\alpha_{\omega}$ below to simulate disk arrays.

In $\mathrm{LiNbO}_{3}$ the SHG susceptibility $\chi_{2 \omega}^{(2)}$ contains a dominant independent element around ten times larger than the rest of the components (e.g., $\chi_{2 \omega, 333}^{(2)}=-83.4 \mathrm{pm} / \mathrm{V}$ reported in Ref. ${ }^{64}$ at $1058 \mathrm{~nm}$ wavelength, although the precise value depends on the exact concentration of $\mathrm{Li}$ and $\mathrm{Nb}$ ). We consider this direction to be parallel to the incident electric field (i.e., perpendicular to the disk rotational axis). Then, we simplify the computation of the nonlinear FoM for SHG $\left(\mathcal{F}_{2 \omega}^{(2)}\right)$ by only including this field component. As we show in Fig. 3c, prominent features associated with the resonances in the linear absorption/polarizability spectra also appear in the FoM for SHG, although the resonance enhancement is rather low.

Nonlinear enhancement driven by lattice resonances in 2D particle arrays. Apart from plasmons and dielectric cavities, lattice resonances provide an efficient way of reaching high-field enhancements, producing for example full reflection even when arbitrarily weak scatterers are considered, ${ }^{65}$ a result that is only made possible by the divergent field enhancement that accompanies the constructive interference among elements of the array. ${ }^{66}$ Here, we investigate the enhancement of optical nonlinearities that can be achieved by arranging individual resonators into a 2D array. As a concrete example, we consider SHG from $\mathrm{LiNbO}_{3}$ nanodisks such as those studied in the previous section, organized in a square array of period $a$ and illuminated by light of intensity $I_{\omega}$ impinging at normal incidence on the plane of the array, as illustrated schematically in Fig. 4a.

For simplicity, we represent the disks through their linear polarizabilities $\alpha_{\omega}^{(1)}$ and $\alpha_{2 \omega}^{(1)}$ at frequencies $\omega$ and $2 \omega$, which is a good approximation for $D \ll a$. Then, through a 

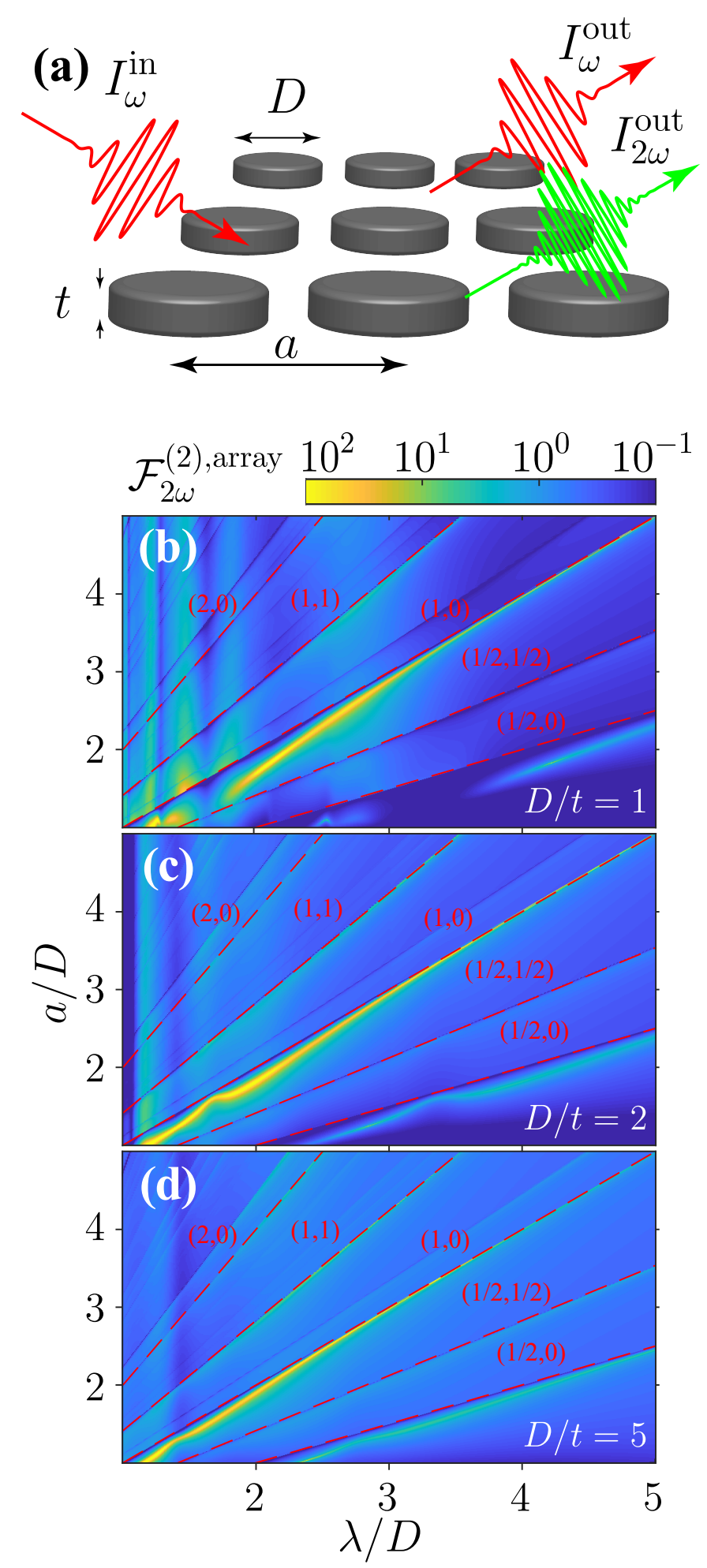

Figure 4: Intrinsic SHG enhancement produced by lattice resonances. (a) We consider a 2D periodic square-lattice array of $\mathrm{LiNbO}_{3}$ disks (period $a$ ), for which we simulate the SHG intensity $I_{2 \omega}$ in resonse to normally-incident light of intensity $I_{\omega}$. (b-d) SHG figure of merit for disks with the same three different aspect ratios $D / t$ as considered in Fig. 3, as a function of incident light wavelength and light period, both normalized to the disk diameter $D$. 
straightforward extension of the methods presented in Refs., ${ }^{52,67}$ we can express the FoM for SHG in the 2D array as

$$
\mathcal{F}_{2 \omega}^{(2), \text { array }}=\frac{\mathcal{F}_{2 \omega}^{(2)}}{\left|\left(1-\alpha_{2 \omega}^{(1)} G_{2 \omega}\right)\left(1-\alpha_{\omega}^{(1)} G_{\omega}\right)^{2}\right|},
$$

where $\mathcal{F}_{2 \omega}^{(2)}$ is the FoM calculated above in for individual disks. In Eq. $(4), G_{\omega}=\sum_{\mathbf{R} \neq 0}\left(\omega^{2} / c^{2}+\right.$ $\left.\partial_{x x}^{2}\right) \mathrm{e}^{\mathrm{i} \omega R / c} / R$ is a sum over lattice vectors $\mathbf{R}$ that accounts for the dipole-dipole interactions between the nanodisks at frequency $\omega$. This sum corresponds to dipoles of the same magnitude, as imposed by the condition of normal incidence. Unfortunately, it is poorly convergent in real space, so it needs to be performed using Ewald's method, which we have implemented following the approach developed by Kambe for $2 \mathrm{D}$ arrays. ${ }^{68}$

The FoM for SHG in the 2D array is presented in Fig. 4b-d as calculated from Eq. (4), with each contour plot corresponding to a specific nanodisk aspect ratio $D / t$. For the sake of clarity, the color scales are saturated to 10, although in each case the maximum value is indicated on the contour plot. We observe regions of increased effective nonlinear response indicated by several linear features, which correspond to the conditions $\operatorname{Re}\left\{1 / \alpha_{\omega}^{(1)}-G_{\omega}\right\}=0$ and $\operatorname{Re}\left\{1 / \alpha_{2 \omega}^{(1)}-G_{2 \omega}\right\}=0$ (see Eq. (4)). Given the small values of the polarizability in units of $D^{3}$ (see Fig. 3b), these conditions can only be met near the divergences of the lattice sum, which arise when the period-to-wavelength ratio satisfies $a / \lambda=\sqrt{m^{2}+n^{2}}$ (dashed lines in Fig. 4b-d), where $(m, n)$ run over diffraction orders; these are the so called Wood anomalies. ${ }^{69}$ We note that the actual maxima are slightly displaced with respect to the Wood anomalies as a result of the finite value of the polarizability.

In contrast to the isolated disks, higher aspect ratios (i.e., thinner disks) offer larger enhancements in the array, an effect that can be attributed to the noted increase in resonant field enhancement with decreasing particle size. ${ }^{65}$ The observed enhancement in SHG with respect to individual disks is significant, reaching values comparable to those obtained with wire plasmons. In principle, the SHG enhancement can reach arbitrarily large values for 
increasingly smaller disks, ${ }^{52}$ only limited by fabrication imperfections, and ultimately by absorption in the material, although in practice the resonances also become narrower, as already observed in the series of plots shown in Fig. 4b-d.

An analytical estimate of the enhancement due to the array can be obtained through a direct extension of the theory in Ref. ${ }^{52}$ for the $(1,0)$ resonance, assuming small disks $(D \ll \lambda)$ : considering Eq. (4), we first note that $\operatorname{Re}\left\{G_{\omega}\right\}$ exhibits a divergence that is perfectly canceled by $\operatorname{Re}\left\{1 / \alpha_{\omega}^{(1)}\right\}$ (regardless how small the polarizability becomes); additionally, we note the exact analytical result $\operatorname{Im}\left\{G_{\omega}\right\}=2 \pi \omega / c a^{2}-2 \omega^{3} / 3 c^{3}$, in which the second term is also perfectly cancelled by $\operatorname{Im}\left\{1 / \alpha_{\omega}^{(1)}\right\}=-2 \omega^{3} / 3 c^{3}$, valid in the absence of inelastic losses with $D \ll \lambda$; finally, under these conditions there is a divergence of $G_{2 \omega}$ due to the $(2,0)$ diffraction order, although it does not necessarily lead to a resonance, as this would imply a coincidental cancellation of the real part of $G_{2 \omega}$ with $\operatorname{Re}\left\{1 / \alpha_{2 \omega}^{(1)}\right\}$. Putting these elements together, we quantify the enhancement near the $(1,0)$ resonance as

$$
\frac{\mathcal{F}_{2 \omega}^{(2) \text {,array }}}{\mathcal{F}_{2 \omega}^{(2)}}=\frac{1}{16 \pi^{4}} \frac{\lambda^{6}}{\left|\alpha_{\omega}^{(1)}\right|^{2}} .
$$

Although this argument implies that it is in principle possible to obtain arbitrarily large SHG enhancement through lattice resonances, this effect is associated with a strong lineshape narrowing that renders them extremely sensitive to defects and inelastic absorption.

Interestingly, the second-harmonic signal at frequency $2 \omega$ can also resonate with the lattice, an effect that is clearly visible in Fig. 4d through the two intense bands below the $(1,0)$ lattice resonance at frequency $\omega$ (therefore labeled by semi-integer numbers $(m, n))$. Actually, these bands fulfill the condition $2 a / \lambda=\sqrt{m^{2}+n^{2}}$ for $(m, n)=(1,0)$ and $(1,1)$, and therefore, they are resonant at the SHG frequency, but not at the fundamental one. 


\section{Conclusions}

By employing a general, material- and geometry-independent definition of the FoM for the enhancement of optical nonlinearities that can be achieved using resonant cavities, we have been able to compare the nonlinear yield for various structures. From a general perspective, we need enhanced fields, for which we can think of complex hybrid structures combining plasmonic, dielectric, and lattice resonances (e.g., cascaded resonances, ${ }^{70}$ nanoparticles coupled to tapered waveguide focusers ${ }^{71}$ and electronic transitions in semiconductor quantum wells, ${ }^{72}$ plasmons and lattice resonances coupled to Fabry-Perot cavities ${ }^{73}$ light trapped in the continuum $\left.{ }^{74}\right)$. The present work provides an assessment of common types of simple cavities and a methodology for comparing their efficiency that can be readily extended to complex hybrid scenarios. Specifically, we have studied the enhancement offered by localized plasmons in Ag nanorods, Mie resonances in dielectric spheres and nanodisks, and lattice resonances in 2D arrays, which are experimentally feasible geometries. Based on our quantification of the nonlinear response, we have shown that Ag nanorods can dramatically boost the nonlinear response in 2D materials, while Mie resonances in dielectric spheres provide more modest enhancements. Additionally, compared to an isolated nanoparticle, we predict that 2D arrays of dielectric nanoparticles produce order-of-magnitude increases in the

nonlinear response. The used FoM provides an efficient tool to explore the dependence of the nonlinear response enhancement by optical resonances on the size-to-wavelength ratio, composition, and geometry, thus facilitating the design of structures with optimal nonlinear yields.

\section{Acknowledgments}

This work has been supported in part by the Spanish MINECO (MAT2014-59096-P and SEV2015-0522), the European Commission (Graphene Flagship 696656), Agència de Gestió d'Ajuts Universitaris i de Recerca (AGAUR) (2014-SGR-1400), the Catalan CERCA Pro- 
gram, and Fundació Privada Cellex.

\section{References}

1. Garmire, E. Nonlinear Optics in Daily Life. Opt. Express 2013, 21, 30532-30544.

2. Rottwitt, K.; Tidemand-Lichtenberg, P. Nonlinear Optics: Principles and Applications; CRC Press: Florida, 2014; Vol. 3.

3. Singh, S. P.; Singh, N. Nonlinear Effects in Optical Fibers: Origin, Management and Applications. Prog. Electromag. Res. 2007, 73, 249-275.

4. Campagnola, P. J.; Lewis, A.; Loew, L. M. High-Resolution Nonlinear Optical Imaging of Live Cells by Second Harmonic Generation. Biophys. J. 1999, 77, 3341-3349.

5. Dempsey, W. P.; Fraser, S. E.; Pantazis, P. SHG Nanoprobes: Advancing Harmonic Imaging in Biology. Bioessays 2012, 34, 351-360.

6. Staedler, D.; Magouroux, T.; Hadji, R.; Joulaud, C.; Extermann, J.; Schwung, S.; Passemard, S.; Kasparian, C.; Clarke, G.; Gerrmann, M. et al. Harmonic Nanocrystals for Biolabeling: A Survey of Optical Properties and Biocompatibility. ACS Nano 2012, 6, 2542-2549.

7. Mesch, M.; Metzger, B.; Hentschel, M.; Giessen, H. Nonlinear Plasmonic Sensing. Nano Lett. 2016, 16, 3155-3159.

8. Yu, R.; Cox, J. D.; García de Abajo, F. J. Nonlinear Plasmonic Sensing with Nanographene. Phys. Rev. Lett. 2016, 117, 123904.

9. Ippen, E. P. Principles of Passive Mode Locking. Appl. Phys. B-Lasers Opt. 1994, 58, $159-170$.

10. Boyd, R. W. Nonlinear Optics, 3rd ed.; Academic Press: Amsterdam, 2008. 
11. Nasari, H.; Abrishamian, M. S. All-Optical Tunable Notch Filter by Use of Kerr Nonlinearity in the Graphene Microribbon Array. J. Opt. Soc. Am. B 2014, 31, 1691.

12. Marini, A.; Cox, J. D.; García de Abajo, F. J. Theory of Graphene Saturable Absorption. Phys. Rev. B 2017, 95, 125408.

13. Cook, D. J.; Hochstrasser, R. M. Intense Terahertz Pulses by Four-Wave Rectification in Air. Opt. Lett. 2000, 25, 1210-1212.

14. Soljačić, M.; Joannopoulos, J. D. Enhancement of Nonlinear Effects Using Photonic Crystals. Nat. Mater. 2004, 3, 211-219.

15. Ilchenko, V. S.; Savchenkov, A. A.; Matsko, A. B.; Maleki, L. Nonlinear Optics and Crystalline Whispering Gallery Mode Cavities. Phys. Rev. Lett. 2004, 92, 043903.

16. Genevet, P.; Tetienne, J. P.; Gatzogiannis, E.; Blanchard, R.; Kats, M. A.; Scully, M. O.; Capasso, F. Large Enhancement of Nonlinear Optical Phenomena by Plasmonic Nanocavity Gratings. Nano Lett. 2010, 10, 4880-4883.

17. Monat, C.; Corcoran, B.; Pudo, D.; Ebnali-Heidari, M.; Grillet, C.; Pelusi, M. D.; Moss, D. J.; Eggleton, B. J.; White, T. P.; O’Faolain, L. Slow Light Enhanced Nonlinear Optics in Silicon Photonic Crystal Waveguides. IEEE J. Sel. Topics Quantum Electron. 2010, 16, 344-356.

18. Armstrong, J. A.; Bloembergen, N.; Ducuing, J.; Pershan, P. S. Interactions between Light Waves in a Nonlinear Dielectric. Phys. Rev. 1962, 127, 1918.

19. Hobden, M. V. Phase-Matched Second-Harmonic Generation in Biaxial Crystals. J. Appl. Phys. 1967, 38, 4365-4372.

20. Mingaleev, S. F.; Kivshar, Y. S. Nonlinear Transmission and Light Localization in Photonic-Crystal Waveguides. J. Opt. Soc. Am. B 2002, 19, 2241-2249. 
21. Assanto, G.; Peccianti, M.; Conti, C. Nematicons: Optical Spatial Solitons in Nematic Liquid Crystals. Opt. Photon. News 2003, 14, 44-48.

22. Kim, Y.; Johnson, R. C.; Hupp, J. T. Gold Nanoparticle-Based Sensing of Spectroscopically Silent Heavy Metal Ions. Nano Lett. 2001, 1, 165-167.

23. Neely, A.; Perry, C.; Varisli, B.; Singh, A. K.; Arbneshi, T.; Senapati, D.; Kalluri, J. R.; Ray, P. C. Ultrasensitive and Highly Selective Detection of Alzheimer's Disease Biomarker Using Two-Photon Rayleigh Scattering Properties of Gold Nanoparticle. ACS Nano 2009, 3, 2834-2840.

24. Singh, A. K.; Senapati, D.; Wang, S.; Griffin, J.; Neely, A.; Candice, P.; Naylor, K. M.; Varisli, B.; Kalluri, J. R.; Ray, P. C. Gold Nanorod Based Selective Identification of Escherichia coli Bacteria Using Two-Photon Rayleigh Scattering Spectroscopy. ACS Nano 2009, 3, 1906-1912.

25. Lukin, M. D.; Imamoğlu, A. Nonlinear Optics and Quantum Entanglement of Ultraslow Single Photons. Phys. Rev. Lett. 2000, 84, 1419.

26. Braje, D. A.; Balić, V.; Yin, G. Y.; Harris, S. E. Low-Light-Level Nonlinear Optics with Slow Light. Phys. Rev. A 2003, 68, 041801.

27. Kok, P.; Munro, W. J.; Nemoto, K.; Ralph, T. C.; Dowling, J. P.; Milburn, G. J. Linear Optical Quantum Computing with Photonic Qubits. Rev. Mod. Phys. 2007, 79, 135-174.

28. Novoselov, K. S.; Geim, A. K.; Morozov, S. V.; Jiang, D.; Zhang, Y.; Dubonos, S. V.; Grigorieva, I. V.; Firsov, A. A. Electric Field Effect in Atomically Thin Carbon Films. Science 2004, 306, 666-669.

29. Wang, Q. H.; Kalantar-Zadeh, K.; Kis, A.; Coleman, J. N.; Strano, M. S. Electronics and Optoelectronics of Two-Dimensional Transition Metal Dichalcogenides. Nat. Nanotech. 2012, 7, 699-712. 
30. Xia, F.; Wang, H.; Xiao, D.; Dubey, M.; Ramasubramaniam, A. Two-Dimensional Material Nanophotonics. Nat. Photon. 2014, 8, 899-907.

31. Mak, K. F.; Shan, J. Photonics and Optoelectronics of 2D Semiconductor Transition Metal Dichalcogenides. Nat. Photon. 2016, 10, 216-226.

32. Mikhailov, S. A. Quantum Theory of the Third-Order Nonlinear Electrodynamic Effects of Graphene. Phys. Rev. B 2016, 93, 085403.

33. Hendry, E.; Hale, P. J.; Moger, J.; Savchenko, A. K.; Mikhailov, S. A. Coherent Nonlinear Optical Response of Graphene. Phys. Rev. Lett. 2010, 105, 097401.

34. Christensen, T.; Yan, W.; Jauho, A.-P.; Wubs, M.; Mortensen, N. A. Kerr Nonlinearity and Plasmonic Bistability in Graphene Nanoribbons. Phys. Rev. B 2015, 92, 121407(R).

35. Cox, J. D.; Marini, A.; García de Abajo, F. J. Plasmon-Assisted High-Harmonic Generation in Graphene. Nat. Commun. 2017, 8, 14380.

36. Cox, J. D.; Yu, R.; García de Abajo, F. J. Analytical Description of the Nonlinear Plasmonic Response in Nanographene. Phys. Rev. B 2017, 96, 045442.

37. Wang, G.; Marie, X.; Gerber, I.; Amand, T.; Lagarde, D.; Bouet, L.; Vidal, M.; Balocchi, A.; Urbaszek, B. Giant Enhancement of the Optical Second-Harmonic Emission of $\mathrm{WSe}_{2}$ Monolayers by Laser Excitation at Exciton Resonances. Phys. Rev. Lett. 2015, 114, 097403.

38. Le, C. T.; Clark, D. J.; Ullah, F.; Senthilkumar, V.; Jang, J. I.; Sim, Y.; Seong, M. J.; Chung, K. H.; Park, H.; Kim, Y. S. Nonlinear Optical Characteristics of Monolayer $\mathrm{MoSe}_{2}$. Ann. Physik 2016, 528, 551-559.

39. Malard, L. M.; Alencar, T. V.; Barboza, A. P. M.; Mak, K. F.; de Paula, A. M. Observation of Intense Second Harmonic Generation from $\mathrm{MoS}_{2}$ Atomic Crystals. Phys. Rev. B 2013, 87, 201401. 
40. Kumar, N.; S. Najmaei, Q. C.; Ceballos, F.; Ajayan, P. M.; Lou, J.; Zhao, H. Second Harmonic Microscopy of Monolayer MoS 2 . Phys. Rev. B 2013, 87, 161403.

41. Zeng, H.; Liu, G. B.; Dai, J.; Yan, Y.; Zhu, B.; He, R.; Xie, L.; Xu, S.; Chen, X.; Yao, W. Optical Signature of Symmetry Variations and Spin-Valley Coupling in Atomically Thin Tungsten Dichalcogenides. Sci. Rep. 2013, 1608.

42. Liu, H.; Li, Y.; You, Y. S.; Ghimire, S.; Heinz, T. F.; Reis, D. A. High-Harmonic Generation from an Atomically Thin Semiconductor. Nat. Phys. 2016, 13, 262-265.

43. Maier, S. A. Plasmonics: Fundamentals and Applications; Springer: New York, 2007.

44. Kauranen, M.; Zayats, A. V. Nonlinear Plasmonics. Nat. Photon. 2012, 6, 737-748.

45. Khurgin, J. B.; Sun, G. Plasmonic Enhancement of the Third Order Nonlinear Optical Phenomena: Figures of Merit. Opt. Express 2013, 21, 27460-27480.

46. Smirnova, D.; Kivshar, Y. S. Multipolar Nonlinear Nanophotonics. Optica 2016, 3, $1241-1255$.

47. Yu, R.; Liz-Marzán, L. M.; García de Abajo, F. J. Universal Analytical Modeling of Plasmonic Nanoparticles. Chem. Soc. Rev. 2017, 46, 6710-6724.

48. Yan1, W.; Faggiani, R.; Lalanne, P. Rigorous Modal Analysis of Plasmonic Nanoresonators. arXiv 2017, 0, 1711.05011.

49. Zhao, Q.; Zhou, J.; Zhang, F.; Lippens, D. Mie Resonance-Based Dielectric Metamaterials. Mater. Today 2009, 12, 60-69.

50. Shcherbakov, M. R.; Neshev, D. N.; Hopkins, B.; Shorokhov, A. S.; Staude, I.; MelikGaykazyan, E. V.; Decker, M.; Ezhov, A. A.; Miroshnichenko, A. E.; Brener, I. et al. Enhanced Third-Harmonic Generation in Silicon Nanoparticles Driven by Magnetic Response. Nano Lett. 2014, 14, 6488-6492. 
51. Saman, J.; Zubin, J. All-Dielectric Metamaterials. Nat. Nanotech. 2016, 11, 23-36.

52. García de Abajo, F. J. Colloquium: Light Scattering by Particle and Hole Arrays. Rev. Mod. Phys. 2007, 79, 1267-1290.

53. Hayat, A.; Orenstein, M. Standing-Wave Nonlinear Optics in an Integrated Semiconductor Microcavity. Opt. Lett. 2007, 32, 2864-2866.

54. Hasan, S. B.; Etrich, C.; Filter, R.; Rockstuhl, C.; Lederer, F. Enhancing the Nonlinear Response of Plasmonic Nanowire Antennas by Engineering their Terminations. Phys. Rev. B 2013, 88, 205125.

55. García de Abajo, F. J.; Howie, A. Retarded Field Calculation of Electron Energy Loss in Inhomogeneous Dielectrics. Phys. Rev. B 2002, 65, 115418.

56. Johnson, P. B.; Christy, R. W. Optical Constants of the Noble Metals. Phys. Rev. B 1972, 6, 4370-4379.

57. Mayer, M.; Scarabelli, L.; March, K.; Altantzis, T.; Tebbe, M.; Kociak, M.; Bals, S.; García de Abajo, F. J.; Fery, A.; Liz-Marzán, L. M. Controlled Living Nanowire Growth: Precise Control over the Morphology and Optical Properties of AgAuAg Bimetallic Nanowires. Nano Lett. 2015, 15, 5427-5437.

58. Novotny, L. Effective Wavelength Scaling for Optical Antennas. Phys. Rev. Lett. 2007, 98, 266802.

59. Novotny, L.; Hecht, B. Principles of Nano-Optics; Cambridge University Press: New York, 2006.

60. Mie, G. Beiträge zur Optik Trüber Medien, Speziell Kolloidaler Metallösungen. Ann. Phys. (Leipzig) 1908, 330, 377-445. 
61. García-Etxarri, A.; Gómez-Medina, R.; Froufe-Pérez, L. S.; López, C.; Chantada, L.; Scheffold, E.; Aizpurua, J.; Nieto-Vesperinas, M.; Sáenz, J. J. Strong Magnetic Response of Submicron Silicon Particles in the Infrared. Opt. Express 2011, 19, 4815-4826.

62. Weis, R. S.; Gaylord, T. K. Lithium Niobate: Summary of Physical Properties and Crystal Structure. Appl. Phys. A-Mater. Sci. Process. 1985, 37, 191-203.

63. Arizmendi, L. Photonic Applications of Lithium Niobate Crystals. Phys. Stat. Sol. A 2004, 201, 253-283.

64. Dmitriev, V. G.; Gurzadyan, G. G.; Nikogosyan, D. N. Handbook of Nonlinear Optical Crystals, 3rd ed.; Springer-Verlag: Berlin, 1999; Vol. 64.

65. García de Abajo, F. J.; Gómez-Medina, R.; Sáenz, J. J. Full Transmission through Perfect-Conductor Subwavelength Hole Arrays. Phys. Rev. E 2005, 72, 016608.

66. Lord Rayleigh, Note on the Remarkable Case of Diffraction Spectra Described by Prof. Wood. Philos. Mag. 1907, 14, 60-65.

67. Thongrattanasiri, S.; Koppens, F. H. L.; García de Abajo, F. J. Complete Optical Absorption in Periodically Patterned Graphene. Phys. Rev. Lett. 2012, 108, 047401.

68. Kambe, K. Theory of Low-Energy Electron Diffraction. I. Application of the Cellular Method of Monatomic Layers. Z. Naturforsch. A 1967, 22, 322.

69. Fano, U. Some Theoretical Considerations on Anomalous Diffraction Gratings. Phys. Rev. 1936, 50, 573-573.

70. Li, K. R.; Stockman, M. I.; Bergman, D. J. Self-Similar Chain of Metal Nanospheres as an Efficient Nanolens. Phys. Rev. Lett. 2003, 91, 227402.

71. Stockman, M. I. Nanofocusing of Optical Energy in Tapered Plasmonic Waveguides. Phys. Rev. Lett. 2004, 93, 137404. 
72. Lee, J.; Tymchenko, M.; Argyropoulos, C.; Chen, P.-Y.; Lu, F.; Demmerle, F.; Boehm, G.; Amann, M.-C.; Alù, A.; Belkin, M. A. Giant Nonlinear Response from Plasmonic Metasurfaces Coupled to Intersubband Transitions. Nature 2014, 511, 6569.

73. Yu, R.; Pruneri, V.; García de Abajo, F. J. Resonant Visible Light Modulation with Graphene. ACS Photon. 2015, 2, 550-558.

74. Hsu, C. W.; Zhen, B.; Lee, J.; Chua, S.-L.; Johnson, S. G.; Joannopoulos, J. D.; Soljačić, M. Observation of Trapped Light within the Radiation Continuum. Nature 2013, 499, 188-191. 